\title{
Applicator Device
}

National Cancer Institute

\section{Source}

National Cancer Institute. Applicator Device. NCI Thesaurus. Code C50341.

A device designed to transfer a substance to a surface. 